\title{
Intelligent Tool Management Strategies for Automated Manufacturing Systems
}

\author{
D. Ganeshwar Rao ${ }^{*}$, C. Patvardhan, Ranjit Singh \\ Faculty of Engineering, Dayalbagh Educational Institute, Dayalbagh, Agra, India \\ E-mail:"dgrao.dei@gmail.com \\ Received July 9, 2011; revised July 30, 2011; accepted August 7, 2011
}

\begin{abstract}
With the increase in automation and use of computer control in machine tools, the number of cutting tools per machining setup is on the increase. On one hand, such multi-tool setups offer the advantages of reduced down-time and cost of production and require less space and in-process inventory, and on the other hand, require proper tool management for economic operations. A number of strategies have been devised to solve the tool selection problems and a number of tool replacement policies have been proposed in the past. These strategies have been solved in isolation, whereas, a comprehensive algorithm for proper selection of tools out of those available in the tool magazine for performing operation and for replacement of tools on failure/wear is necessary. In this paper, taking cue from the computer memory management policies, four tool selection strategies have been presented and their performance in tandem with various tool replacement policies has been studied. The effect of important parameters such as reduction of tool life due to regrinding, limited number of regrindings, catastrophic failures etc. have been considered. Cost has been computed for each combination of tool selection and replacement policy. Also, the number of machine stoppages has been worked out in each case. The results indicate that the combination of various selection strategies with suitable replacement policies affects the overall cost.
\end{abstract}

Keywords: Tool Management, Tool Selection, Tool Replacement, Multi-Tool Setup

\section{Introduction}

The present manufacturing industry requires producing a large variety of complex components in small batch sizes and in a cost effective manner. At the same time the number of tools to be magazined has increased, particularly in automated machining centres. It is not unusual for there to be over 300 different tools in a direct storage unit at a machining centre. Such multi-tool setups offer the advantages of reduced time and cost of production and require less space and in-process inventory. With the above developments, the tool management task has become substantially more demanding because the increase in number of tools per setup requires a better tool reliability management, as wearing/failure of any one tool renders the whole system non-operational. As the number of tools in the machining setup increases, the frequency of replacement due to wearing out of tool and due to failure of tool also increases. A tool management system adopted should be such as to make the right tool available at the right time in proper sequence for processing a job while keeping costs down to a reasonable limit. Hence, it becomes necessary to devise strategies which would select the right tool out of those available in the tool magazine of the machining centre [1-3].

In a traditional machining environment, a skilled operator, in liaison with the tool store personnel monitors, maintains, and replenishes the small number of tools associated with each machine tool. The expertise of the machine operator is the determinant factor in ensuring that correct tools are used for each operation and the tools are replaced before they completely wear out and damage the work piece. This working pattern is no longer acceptable in a modern machine shop equipped with the $\mathrm{CNC}$ ma-chines, as a greater variety, and a large number of tools are used to machine components on these machines and the task of determining the correct tool out of the alternate tools available, and task of replacing the tool on failure or before failure, is too complicated to be left to the machine operator. Also, a large number of machine stoppages may be caused due to incorrect tool selection and replacement. The tasks of tool selection for performing the operations, 
and tool replacement decisions are complex and require development of sophisticated programs which should be supported by databases containing information on the manufacturing resources and company-specific machining practices [4].

\section{Literature Review}

Tool management problems have been attempted by researchers since the turn of the century. The detailed survey of literature is as follows.

\subsection{Studies on Tool Replacement}

A thorough review of the solution methodologies related to tool replacement can be found in McCullough [5], Armarego and Brown [6], Pa'sko [7], Batra and Barash [8], Bao [9], LaCommare et al. [10], Sharit and Elhence [11], Zhou et al. [12] and Tak [13]. As has been mentioned by Zhou et al. [12], the various authors have classified tool replacement strategies in different ways. Most probabilistic optimization models are based on tool life distribution [10,14]. A major development in the process of computer integration of automated manufacturing systems has been the implementation of automated tool replacement $[15,16]$. A complete Tool Replacement Strategy specifies a tool change schedule based upon the economic service lives of tools and a control policy regarding unscheduled tool changes following breakage. The most realistic replacement strategies have considered the distributed nature of tool lives under actual machining parameters, as well as the option to change several tools once one fails $[9,10]$, rather than considering only expected lives and single tool replacement [5,6]. All of these tool replacement studies have considered one machine in isolation. Sharit and Elhence [11] have gone beyond the single machine model to examine tool replacement strategy at the system level. Rather than proposing an automated, optimizing strategy, their study emphasizes the limitations of both human and computer at making the tradeoff between economic tool replacement costs and system throughput in a real-time, dynamic environment. Zhou et al. [12] have proposed an optimization model for tool replacement based on tool wear status. The model is capable of utilizing tool wear status in determining an optimal replacement policy. They have mentioned three types of tool replacement strategies employed in the industry: 1) scheduled tool replacement, 2) preventive planned tool replacement, and 3) failure tool replacement. Under the first strategy, the cutting tool is replaced either at pre-scheduled time or upon failure whichever is earlier. The second is also similar, except that it is based on the number of finished workpieces. The third strategy is to use the tool until failure. Research has been done to find the optimum pre-established time or lot size for replacement and to compare the different strategies [10].

Two broad categories of the tool replacement policies, viz. unscheduled tool replacement schemes and scheduled tool replacement schemes have been mentioned in the literature. Under unscheduled tool replacement scheme, Series Tool Replacement and Parallel Tool Replacement have been suggested [8]. Under scheduled tool replacement schemes, Individual Tool Replacement [7], Group Tool Replacement [8], Skip Schedule Group Tool Replacement and Sub Group Tool Replacement [13] have been suggested. Tak [13] has suggested the Dynamic Tool Replacement policy, under which every tool failure, which essentially results in machine interruption, is utilized as an opportunity for evaluating every tool of the setup for its effective utilization and reliability. Under this strategy, whenever a tool fails, all the tools in the magazine are evaluated for the useful lives that they have lived and the tools exceeding their warning limits are also replaced along with the tool which has failed. Hedin et al. [17] have investigated static and dynamic tooling policies and presented a comparison between the two in context of a general flexible manufacturing system. Currently, many tool replacement models are deficient in that they ignore the relationship between the processing rates and the tool replacement policy.

\subsection{Studies on Tool Selection}

Major research efforts in the area of Tool Selection started in the early 80's. A review of solution methodologies for optimum tool selection can be found in Cederqvist [18], Giusti et al. [19], Chen et al. [20], Syan [21], Domazet [22], Maropoulos [23], Hinduja and Barrow [24], Eversheim et al. [2], Zhang and Hinduja [4], Hedin et al. [17]. The task of selecting cutting tools which are not only functionally correct but also optimum, is a complex one. Cederqvist [18] has suggested that when a new batch is planned, the number of cutting edges required for each tool in the setup can be calculated and a complete set of block tool heads can then be prepared and sent out to the machine. Some researchers viz. Giusti et al. [19], Syan [21] have developed expert systems wherein the technological knowledge is represented as production rules which are consulted when selecting a tool for a given operation. Chen et al. [20] have adopted a heuristic-deterministic approach to reduce the computing time to determine the optimum tooling for rough turning operations. Domazet [22] has proposed a hybrid approach to automatically select turning tools; the selection is done in stages and for those stages which require 
special knowledge and expertise, a non-algorithmic method is followed. Maropoulos [23] has used an algorithm approach to automatically determine tools for rough and finish turning operations. Hinduja and Barrow [24] have suggested an automatic interactive system. In the interactive part, the user is guided by the system towards the parameters of the optimum tool. Eversheim et al. [2] have reiterated the importance of tool selection in a modern manufacturing environment. They have proposed an integrated method for tool selection on the basis of manufacturing features. Zhang and Hinduja [4] have proposed automatic generation of a tool set for a given batch of components, the optimization criterion being either the minimum machining cost or minimum number of machine stoppages or a combination of both. Hong-Bae Jun et al. [25] have considered a tool provisioning problem in a flexible manufacturing system (FMS) with an automatic tool transporter. Their study determines the number of copies of each tool type for a limited budget with the objective of minimizing makespan. Two heuristic algorithms have been proposed. One is a composite search algorithm based on two greedy search methods, and the other is a search algorithm in which numbers of tool copies are determined based on tool groupings. In both algorithms, simulation results are used to find search directions. Mözbayrak et al. [26] have addressed the design of an integrated tool management system for flexible machining facilities (FMFs). Modules with functions ranging from issuing tools according to a tooling strategy to diagnosing system operation have been developed and integrated around a centralized manufacturing database to guarantee streamlined manufacture. Selim Akturk M. et al. [27] have shown that there is a critical interface between the lot sizing and tool management decisions, and these two problems cannot be viewed in isolation. They have proposed the alternative algorithms to solve lot sizing, tool allocation and machining conditions optimization problems simultaneously. Svinjarević G. et al. [28] have studied the controlled testing and analysis in every phase of tool management in departments and other services which are directly involved in the tool management system to reduce stock and costs. They have identified some disadvantages and given a few suggestions for the improvement in the tool management system. Haslina Arshad et al. [29] have introduced a virtual cutting tool management system to reduce or eventually solve many of the tool management problems. It has the capability of choosing the right cutting tool from a virtual cutting tool catalogue. Their system provides the virtual selection process for cutting tool and a virtual milling process.

\section{Problem Definition}

The above survey of literature reveals that several researchers have attempted different tool selection schemes and tool replacement strategies, but most of the above models, except for a few, developed for optimizing tool replacement, do not consider the actual status of the cutting tool. From the above review, it is clear that research efforts have been mainly directed towards the selection of optimum tooling for a single machining operation. Also, the possibility of adjusting the wear rates of individual tools and synchronizing tool changes due to wear/failure in order to reduce the number of machine stoppages has been suggested. Also, investigations have been carried out in the past using simulation strategies to find out the best strategy under different operating situations. However, it is necessary to investigate how each selection policy performs in tandem with different replacement policies. The reason is that some tool selection strategy might be better in combination with a particular replacement strategy but may prove to be worse with other replacement strategies.

The focus of this paper is to evaluate the performance of identified tool selection policies in tandem with the different tool replacement strategies. In developing a realistic simulation model for evaluating various tool selection and replacement policies, it is very important to consider various factors such as remaining tool life of partially used tools, catastrophic failure of the tools, number of times the tool has been reground, reduction in tool life due to regrinding, applicability of tools for various operations, operations-profile etc. In the present work, a simulation model has been developed to investigate the most appropriate tool selection policy with each replacement strategy considering the above mentioned factors.

\section{Adaptations for Tool Selection and Replacement}

The strategies considered for tool selection and replacement for developing the simulation model have been explained in the following sections.

\subsection{Tool Selection Strategies}

The goal of any tool management system is to make the right tool available at the right time in the right sequence for processing a job. To achieve this goal, the selection of the right tool is very important as there may be a number of similar tools or different tool types in the magazine with different remaining tool lives, capable of performing the operation. If proper selection of the tool 
is not done then it is possible that a certain tool of one type may not be used at all or may be lying unused for a long time in the tool magazine, even if it has a good amount of remaining tool life. The tool selection logic is very simple to describe. The system must maintain a record of all the tools with different tool lives. Some tools may be having a small value of tool life whereas some tools may have a large value of tool life. When a tool request is made, the tool magazine should be searched for the type of tool required and the tool life required. Since the magazine has tools with different tool lives, a procedure for selecting right tool is needed for the optimum utilization of the tools.

In computer systems, memory management is performed in which a system maintains a list of all the blocks of memory. Some of these blocks are free at any time and some currently allocated to a user. When an allocation request is made, the system must locate a free block of memory of sufficient size and allocate all or part of it. In case only a portion of a free block is to be allocated, the allocation is made from the bottom of the block. When a re-allocation request is made, the system must recover the re-allocated block of memory. In addition, the system should be able to find adjacent free blocks of memory and combine them into a single large block, to maximize the probability of being able to satisfy a large allocation request. For this reason, a number of memory management systems have been devised for different applications. Some of the most common are known by the name First Fit, Next Fit, Best Fit and Worst Fit. These policies have been adapted here for selecting the tools from the tool magazine for performing various operations on a machining centre. The adaptation of the policies in context with the tools selection is explained in the following paragraphs.

\section{1) First Fit}

It is a very simple scheme. The available tools are placed in the magazine in a random manner irrespective of their remaining lives. When a tool request is received, the magazine is searched for the first tool with remaining life, large enough to satisfy the request, and the tool is used for processing the requesting job.

\section{2) Next Fit}

In the First Fit scheme, the search for the tool having sufficient remaining life to serve the request always begins from the starting position of the magazine. Consequently, it requires longer time for search and also, all the tools with smaller tool life tend to collect at the beginning. Hence, it is necessary to examine several tools before allocating a tool for processing. A modification to the first fit strategy is to start a search for a suitable tool at the position where the previous search ended. This approach causes the decrease in search time and also, tends to distribute tools with lesser tool life uniformly over the entire magazine rather than concentrating them near the front. This approach is named Next Fit scheme.

\section{3) Best Fit}

The Best Fit approach is to search the entire magazine for the tool with the smallest tool life which satisfies the request. This approach tends to save the tools having larger tool lives until they are needed to satisfy a larger request.

\section{4) Worst Fit}

In this approach, the entire magazine is searched for the tool with largest tool life that satisfies the request. The idea is that the tool life remaining after processing the current request is large enough to process another request. However, this approach tends to generate large number of tools with very small tool lives that are inadequate to satisfy most subsequent requests.

\subsection{Tool Replacement Strategies}

A number of tool replacement schemes have been suggested in the past. Tool replacement has its own importance in the field of manufacturing. If a tool is replaced too early, the remaining tool capacity is lost and too frequent changes take place. On the other hand, if a tool is replaced too late, the probability of tool failure goes high. Since the proposed tool selection schemes are based on the remaining tool life of the tool, the same factor will be considered for tool replacement also. Out of the available schemes in the literature, the following have been adapted in the present work:

\section{1) Single Tool Replacement}

In this replacement scheme, tools are continuously monitored and as and when a tool exceeds its warning limit or fails due to some other reason, it is replaced. This results in full utilization of tool life but results in higher down-time cost.

\section{2) Multi Tool Replacement}

Under this strategy, whenever a tool in the setup exceeds its warning limit or becomes unusable due to failure, all the tools of the setup are replaced. Such a Strategy may result in lower down-time cost but the tools are not utilized to their capacity and hence tooling cost goes much higher.

\section{3) Dynamic Tool Replacement}

Under this scheme, whenever a tool in the setup exceeds its warning limit or becomes unusable due to failure, every other tool of the setup is evaluated for useful life which it has lived. If this life exceeds the critical life of the tool, this tool is also replaced along with the tool which has failed.

\section{Methodology}

A simulation model has been developed to investigate 
the most appropriate tool replacement policy with several tool selection schemes. All the relevant information about the different tools such as, tool material, different operations that can be performed by the tool, maximum tool life, cost of the tool, cost of regrinding, warning limit of tool etc. has been stored in a tool database. Also, the cutting parameters like depth of cut, cutting speed, feed, etc. for different combinations of tool and workpiece material have been stored. The relevant data has been collected from standard hand-books. Several data sets have been created and in each data set operations to be performed, tool material, workpiece material and the type of surface finish required are stored. In each simulation run, the user has to specify the data set and tool selection and replacement policy to be tried. For each operation, the software calculates the tool life required using standard Taylor's tool life equations taking feed, speed, and depth of cut into consideration. The tools are selected for the operation using the policy specified and the relevant data stored. For all the cases the number of regrindings done, total cost involved, and number of times the machine was down, are computed.

\section{1) Cost Function}

$$
\begin{aligned}
\text { Total Cost }= & \text { Machining Cost }+ \text { Down Time Cost } \\
& + \text { Tool Cost }+ \text { Setter Cost } \\
\text { Total Cost }= & (\text { MC }+ \text { CI })+\text { DT }+ \text { TC } \\
& +(\text { MTRA }+ \text { MTSRA }) * \text { SR }
\end{aligned}
$$

where:

$$
\begin{aligned}
& \text { MC = Machining Cost } \\
& \text { CI = Labour Rate }+ \text { Supervision Charges } \\
& \quad \text { + Interest on investment } \\
& \text { DT }=\text { Down Time } \\
& \text { TC }=\text { Tool Cost } \\
& \text { MTRA = Mean Time for tool replacement and ad- } \\
& \quad \text { justment } \\
& \text { MTSRA = Mean Time for setter arrival } \\
& \text { SR = Setter Rate per Hour } \\
& \text { The following factors have been considered in the } \\
& \text { simulation model: }
\end{aligned}
$$

\section{2) Catastrophic Failure}

A considerable percentage of tool inventory is commonly lost due to sudden and unexpected failure or tool breakage. Therefore this feature has been incorporated in the simulation model, with the assumption that about $5 \%$ of the new tools and $15 \%$ of the reground tools fail due to catastrophic failures.

\section{3) Tool Regrindings}

To prevent tool breakage use of excessively reground tools is avoided. In the simulation model, the number of regrindings of tools has been limited to a certain number, depending on the type of tool. Further, it is considered that after every regrinding, the tool life reduces by a certain percentage. For different tools, this percentage varies.
Also, the number of regrindings permissible for each tool is different.

\section{Computational Experience}

A realistic simulation model developed in the present work, evaluates the performance of identified tool selection policies in combination with the different tool replacement strategies. A large number of data sets consisting of the details of different types of tools have been generated randomly. The realistic values of factors such as remaining tool life of partially used tools, catastrophic failure of the tools, number of times the tool has been reground, reduction in tool life due to regrinding, applicability of tools for various operations, and operationsprofile have also been generated and incorporated in the model.

In the model, the four tool selection strategies viz. First Fit, Next Fit, Best Fit and Worst Fit against each tool replacement policy viz. Single Tool Replacement, Multi Tool Replacement and Dynamic Tool Replacement have been considered.

For each data set, the extensive simulations have been carried out and the total cost involved and the number of times the machine was down due to change of tool have been computed. The results have been tabulated in Tables 1 and 2 . The results indicate the combination of most appropriate 'Replacement Policy' with the appropriate "selection policy" for obtaining minimum cost for each data set. Also, the number of stoppages of machine is obtained for each combination of tool replacement and selection strategy. Figures 1-3 show a comparison of results in the form of bar charts clearly indicating the trend for some of the data sets used in the simulation.

\section{Conclusions}

The number of stoppages of machine due to wearing or failure of tool plays an important role in increasing the cost of production. In the present work, the tool selection and replacement strategies have been identified. The simulation model developed selects the best combination of these strategies for minimizing stoppages and the cost of production. It takes into consideration the effect of important parameters such as reduction of tool life due to regrinding, limited number of regrindings, a catastrophic failure etc.

From the results obtained, it can be concluded that the combination of various selections policies and replacement policies affects the overall cost. The investigations reveal that the total cost involved is lowest in the case of Dynamic Replacement Policy in tandem with the worst fit approach. However, considering the limitations of the 
Table 1. Total cost with different strategies for each dataset.

\begin{tabular}{ccccccccc}
\hline \multirow{2}{*}{ Replacement Policy } & Selection & \multicolumn{9}{c}{ DATA SETS } \\
\cline { 3 - 9 } & Policy & I & II & III & IV & V & VI & VII \\
\hline & First Fit & 12,126 & 5166 & 7790 & 7534 & 4162 & 3255 \\
Series Tool & Next Fit & 11,651 & 4791 & 8132 & 6829 & 4172 & 3631 & 8581 \\
Replacement Policy & Best Fit & 13,246 & 8816 & 10,702 & 10,834 & 7472 & 5101 & 10,406 \\
Worst Fit & 11,501 & 4466 & 7432 & 6829 & 3451 & 2546 & 7111 \\
\hline Parallel Tool & First Fit & 13,031 & 4861 & 9757 & 8269 & 3887 & 3406 & 8901 \\
Replacement Policy & Next Fit & 12,691 & 5036 & 10,742 & 9724 & 4062 & 3756 & 9776 \\
& Best Fit & 13,911 & 9216 & 9727 & 11,084 & 9632 & 5231 & 12,236 \\
& Worst Fit & 12,261 & 4531 & 8767 & 8369 & 3457 & 2546 & 8296 \\
\hline \multirow{2}{*}{ Dynamic Tool } & First Fit & 11,801 & 4831 & 7782 & 7534 & 3837 & 3240 & 7880 \\
Replacement Policy & Next Fit & 11,551 & 4791 & 7762 & 6864 & 3882 & 3306 & 8166 \\
& Best Fit & 12,646 & 7866 & 9667 & 10,254 & 7987 & 4526 & 10,131 \\
& Worst Fit & 11,501 & 4466 & 7432 & 6829 & 3451 & 2546 \\
\hline
\end{tabular}

Table 2. Number of times the machine was down.

\begin{tabular}{|c|c|c|c|c|c|c|c|c|}
\hline \multirow{2}{*}{ Replacement Policy } & \multirow{2}{*}{$\begin{array}{l}\text { Selection } \\
\text { Policy }\end{array}$} & \multicolumn{7}{|c|}{ DATA SETS } \\
\hline & & I & II & III & IV & $\mathbf{V}$ & VI & VII \\
\hline \multirow{4}{*}{$\begin{array}{c}\text { Series Tool } \\
\text { Replacement Policy }\end{array}$} & First Fit & 8 & 3 & 4 & 4 & 2 & 2 & 4 \\
\hline & Next Fit & 6 & 2 & 5 & 2 & 2 & 3 & 6 \\
\hline & Best Fit & 11 & 13 & 12 & 13 & 11 & 7 & 12 \\
\hline & Worst Fit & 6 & 1 & 3 & 2 & 0 & 0 & 3 \\
\hline \multirow{4}{*}{$\begin{array}{c}\text { Parallel Tool } \\
\text { Replacement Policy }\end{array}$} & First Fit & 9 & 2 & 8 & 5 & 1 & 2 & 7 \\
\hline & Next Fit & 7 & 2 & 8 & 6 & 1 & 2 & 7 \\
\hline & Best Fit & 8 & 10 & 6 & 10 & 13 & 5 & 12 \\
\hline & Worst Fit & 7 & 1 & 6 & 5 & 0 & 0 & 5 \\
\hline \multirow{4}{*}{$\begin{array}{c}\text { Dynamic Tool } \\
\text { Replacement Policy }\end{array}$} & First Fit & 7 & 2 & 4 & 4 & 1 & 2 & 5 \\
\hline & Next Fit & 6 & 2 & 4 & 2 & 1 & 2 & 7 \\
\hline & Best Fit & 9 & 10 & 9 & 11 & 12 & 5 & 11 \\
\hline & Worst Fit & 6 & 1 & 3 & 2 & 0 & 0 & 3 \\
\hline
\end{tabular}

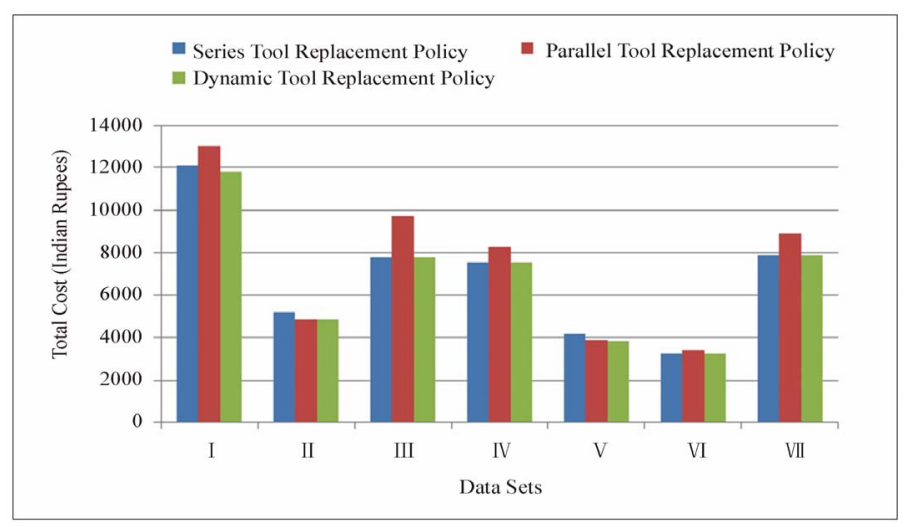

Figure 1. Tool cost on applying first fit tool selection policy with different tool replacement policies.

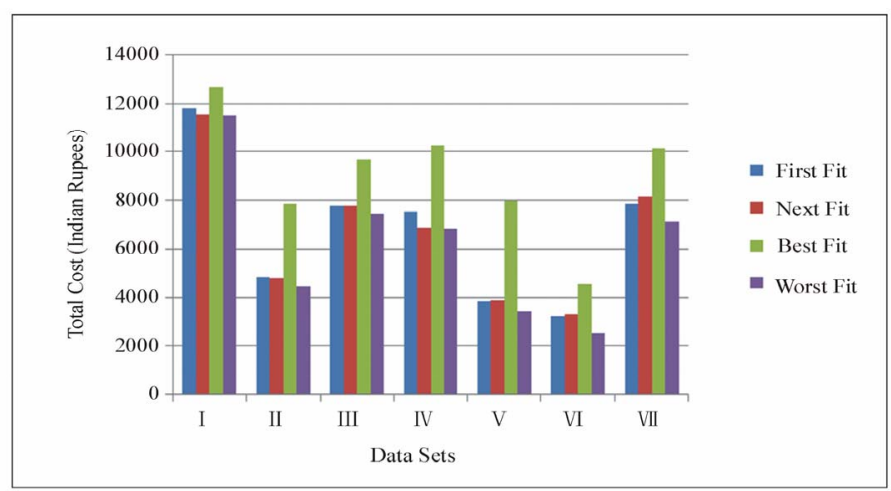

Figure 2. Tool cost on applying dynamic tool replacement policy with four tool selection policies. 


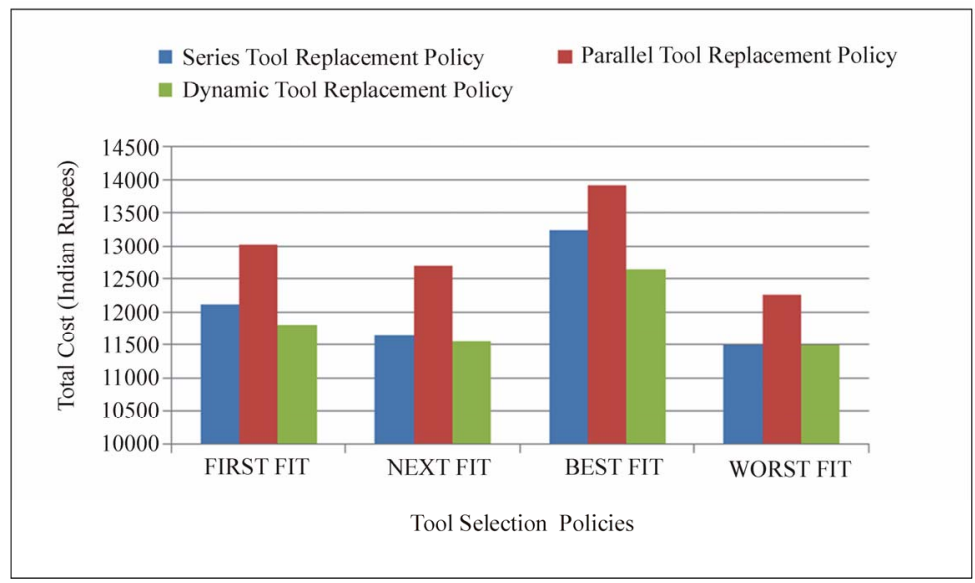

Figure 3. Total cost on applying three tool replacement policies with four tool selection policies on dataset 1.

simulation model, these results cannot be directly generalized to any other data set, but the simulation can be carried out for other data sets also to select the best combination of selection and replacement policies.

\section{References}

[1] P. Tomek, "Tooling Strategies Related to FMS Management," FMS Magazine, Vol. 5, 1986, pp. 102-107.

[2] W. Eversheim, M. Lenhart and B. Katzy, "Information Modelling for Technology Oriented Tool Selection," Annals of the CIRP, Vol. 43, No. 1, 1994, pp. 429-432. doi:10.1016/S0007-8506(07)62246-X

[3] S. Pal, "An Expert System Approach for Scheduling and Tool management in an FMS Environment," Unpublished M.Tech. Dissertation, I.I.T. Kanpur, Kanpur, 1991.

[4] J. H. Zhang and S. Hinduja, "Determination of the Optimum Tool Set for a Given Batch of Turned Components," Annals of the CIRP, Vol. 44, No. 1, 1995, pp. 445-450. doi:10.1016/S0007-8506(07)62360-9

[5] E. M. McCullough, "Economics of Multi-Tool Lathe Operations," American Society of Mechanical Engineers, New York, 1963, pp. 402-404.

[6] E. J. A. Armarego and R. H. Brown, "The Machining of Metals," Prentice Hall, Inc., Englewood Cliffs, 1969.

[7] N. I. Pa'sko, "Optimization of Multi-Tool Setups with Tool Life Scatter," Russian Engineering Journal, Vol. 80, No. 9, 1970, pp. 82-85.

[8] J. L. Batra and M. M. Barash, "Automated Computerized Optimization of Multi-Spindle Drilling with Probabilistic Tool Life," Proceedings of 14th International Machine Tool Design and Research Conference, Manchester, 1973, pp. 125-136.

[9] H. Bao, "Applications of Dynamic Programming to Optimize Tool Replacement Schedules for Multi-Tool Operations Involving Distributed Tool Lives," ASME Journal of Mechanical Design, Vol. 102, 1980, pp. 446-451. doi:10.1115/1.3254767

[10] U. LaCommare, L. Diega, S. Nota and A. Passannante, “Optimum Tool Replacement Policies with Penalty Cost for Un- foreseen Tool Failures," International Journal of Production Research, Vol. 23, 1983, pp. 237-243.

[11] J. Sharit, and S. Elhence, "Computerization of Tool Replacement Decision Making in Flexible Manufacturing Systems: A Human-System Perspective," International Journal of Production Research, Vol. 27, No. 12, 1989, pp. 2027 2039. doi:10.1080/00207548908942672

[12] C. Zhou, J. Chandra and R. Wysk, "Optimal Cutting Tool Replacement Based on Tool Wear Status," International Journal of Production Research, Vol. 28, No. 7, 1990, pp. 1357-1367. doi:10.1080/00207549008942798

[13] S. S. Tak, "Dynamic Tool Replacement Strategy and Its Characteristics," Unpublished Ph.D. Dissertation, University of Jodhpur, Jodhpur, 1989.

[14] S. Noro La Diega and A. Passannanti, "Optimum Tool Replacement Policies with Running-in of the Cutting Edge," CIRP Annals-Manufacturing Technology, Vol. 33, No. 1, 1984, pp. 1-3.

[15] G. D. Crite, R. I. Mills and J. J. Talavage, "PATHSIM, A Modular Simulator for an Automatic Tool Handling System Evaluation in FMS," Journal of Manufacturing System, Vol. 4, No. 1, 1985, pp. 15-28. doi:10.1016/0278-6125(85)90004-4

[16] H. A. ElMaraghy, "Automated Tool Management in Flexible Manufacturing," Journal of Manufacturing Systems, Vol. 4, No. 1, 1985, pp. 1-13. doi:10.1016/0278-6125(85)90003-2

[17] S. R. Hedin, P. R. Philipoom and M. K. Malhotra, "A Comparison of Static and Dynamic Tooling Policies in a General Manufacturing System," IIE Transactions, Vol. 29, No. 1, 1997, pp. 69-80. doi:10.1080/07408179708966313

[18] A. Cederqvist, "Cutting Tool Development for Automation and FMS," Proceedings of 4th International Conference on FMS, Stockholm, 15-17 October 1985, pp. 187-197.

[19] F. Giusti, M. Santochi and G. Dini, "COATS: An Expert Module for Optimal Tool Selection," CIRP Annals-Manufacturing Technology, Vol. 35, No. 1, 1986, pp. 337-340.

[20] S. Chen, S. Hinduja and G. Barrow, "Automatic Selection of Tool for Roughing Operation," International Journal of Machine Tool Manufacturing, Vol. 29, No. 4, 1989, pp. 535553. doi:10.1016/0890-6955(89)90070-9

[21] S. C. Syan, "Selecting Tools Like the Expert," Journal of 
Integrated Manufacturing System, Vol. 1, No. 4, 1990, pp. 187-189. doi:10.1108/EUM0000000002072

[22] D. Domazet, "The Automatic Tool Selection with the Production Rules Matrix Method," CIRP Annals-Manufacturing Technology, Vol. 39, No. 1, 1990, pp. 497-500.

[23] P. G. Maropoulos and S. Hinduja, "Automatic Tool Selection for Rough Turning," International Journal of Production Research, Vol. 29, No. 6, 1991, pp. 1185-1204. doi:10.1080/00207549108930127

[24] S. Hinduja and G. Barrow, "Sits-A Semi-Intelligent Tool Selection System for Turned Components," CIRP AnnalsManufacturing Technology, Vol. 42, No. 1, 1993, pp. 535539.

[25] H.-B. Jun, Y.-D. Kim and H.-W. Suh, "Heuristics for a Tool Provisioning Problem in a Flexible Manufacturing System with an Automatic Tool Transporter," Robotics and Automation, Vol. 15, No, 3, 1999, pp. 488-496.

[26] Mözbayrak, R. B. R. De Souza and R. Bell, "Design of a
Tool Management System for a Flexible Machining Facility," Proceedings of the Institution of Mechanical Engineers, Part B: Journal of Engineering Manufacture, Vol. 215, No. 3, 2001, pp. 353-370.

doi:10.1243/0954405011515424

[27] M. S. Akturk and S. Onen, "Dynamic Lot Sizing and Tool Management in Automated Manufacturing Sytems" Computers \& Operations Research, Vol. 29, No. 8, 2002, pp. 1059-1079. doi:10.1016/S0305-0548(00)00103-9

[28] G. Svinjarević, A. Stoić and J. Kopač, "Implementation of Cutting Tool Management System," Journal of Achievements in Materials and Manufacturing Engineering, Vol. 23, No. 1, 2007.

[29] H. Arshad, R. Hassan, N. Omar and S. Sahran, "Virtual Cutting Tool Management System for Milling Process" International Journal of Computer Science and Networks, Vol. 10 No. 2, 2010. 